Int. J. Morphol.,

30(2):422-424, 2012.

\title{
Estudio Morfológico de los Labios en una Población Mestiza Colombiana
}

\author{
Morphological Study of the Lips in a Colombian Population \\ "Sandra M. Galindo-Ramirez; "Jessica Vargas \& "*Mario Alejandro Ortiz
}

GALINDO-RAMIREZ, S. M.; VARGAS, J.; ORTIZ, M. A. Estudio Morfológico de los labios en una población mestiza colombiana. Int. J. Morphol., 30(2):422-424, 2012.

RESUMEN: El objetivo de esta investigación fue determinar diferencias de dimorfismo sexual de las características de labios de hombre y mujeres mestizos colombianos a través de análisis fotogramétrico. Se evaluaron fotografías frontales de 120 individuos mestizos sin alteraciones congénitas o adquiridas de los labios, tomadas en relación 1:1. A través del uso de puntos antropométricos en la vista frontal, se evaluaron las dimensiones: Grosor del Labio Superior (Sn-Stms), Grosor del bermellon superior (Stms-Ls), Grosor del Labio Inferior (Stmi-Sml), Grosor del bermellon Inferior (Stmi-Li) y Ancho bicomisural (Chd-Chi), con la ayuda del software J image (NIH). El análisis estadístico utilizó la prueba U Mann-Whitney usando el software SPSS 17.0, para determinar diferencias entre los dos sexos en todas las variables. No se encontraron diferencias estadísticamente significativas en la longitud del bermellón del labio superior (Stms-Ls) ni en la longitud del bermellón del labio inferior (Stmi-Li). Se encontraron diferencias estadísticamente significativas ( $<<0,001$ ) en Grosor del Labio Superior (Sn-Stms), Grosor del Labio Inferior (Stmi-Sml) y Ancho bicomisural (Chd-Chi). Estas medidas fueron mayores en individuos mestizos de género masculino. Existe dimorfismo sexual en el ancho bicomisural, longitud de labio superior e inferior en Colombianos Mestizos.

PALABRAS CLAVE: Labio; Dimorfismo sexual; Bermellón.

\section{INTRODUCCIÓN}

$\mathrm{La}$ apariencia de los tejidos blandos faciales refleja la estructura de los tejidos duros subyacentes, por lo que el análisis de la superficie es una herramienta válida para el correcto diagnóstico de la estética facial (Ferrario et al., 2009).

Las normas predeterminadas de proporciones faciales ideales han sido compiladas de manera extensa en la literatura (Peck \& Peck, 1995). Los labios son componentes escenciales en la estética y simetría facial. Estudios antropométricos han mostrado la importancia de medidas morfológicas como el ancho bicomisural y el ancho del bermellón (Peck \& Peck; Sawyer et al., 2009).

Se han descrito múltiples métodos de estudio de las características morfológicas de los labios, los cuales incluyen: antropometría labial a través de impresiones labiales (Ferrario et al.; Chiandussi et al., 2007), resonancia magnética, estereografía tridimensional (Sawyer et al.) y la fotografía clínica (Edler et al., 2011; Davis et al., 2010; Cattoni $\&$ Fernandes, 2009).
La evaluación del perfil y de las características estéticas del paciente es fundamental en el diagnóstico y la determinación del plan de tratamiento en ortodoncia. Sin embargo, la identificación de un patrón ideal no es garantía de la obtención de una cara completamente estética (Scott et al., 2006), En este sentido, es de gran importancia evaluar las diferencias étnicas de la belleza más allá del paradigma de belleza caucásico. Esta evaluación permite determinar las medidas antropométricas adecuadas a los individuos de una etnia determinada (Wong et al., 2010). Así, el objetivo del estudio fue identificar la presencia de dimorfismo sexual en las características morfológicas de los labios de una población mestiza colombiana, a través del análisis morfológico fotográfico frontal.

\section{MATERIAL Y MÉTODO}

Se evaluaron 120 sujetos (60 hombres y 60 mujeres) de la Facultad de Odontología de la Universidad Antonio Nariño que decidieron participar en el estudio. Se obtuvo autorización

* Odontóloga, Universidad Antonio Nariño, Palmira, Colombia.

** Odontólogo, Especialista en Ortodoncia, Maestría en Ciencias Básicas Médicas, Departamento de Morfología, Universidad del Valle, Cali, Colombia. 
previa por parte del Comité Institucional de Ética en Investigación de la Facultad de Odontología. Los criterios de inclusión fueron: individuos sistémicamente sanos, mayores de edad, estudiantes de la Universidad Antonio Nariño Sede Palmira. Se excluyeron individuos que presentaran alteraciones infecciosas, traumáticas o de desarrollo a nivel labial que afectaran la localización de los puntos antropométricos. Los pacientes cumplieron características faciales de individuos mestizos colombianos y debido a la pequeña cantidad de individuos afrocolombianos, las características de la población negra no pudieron ser evaluadas.

A cada individuo se le tomó una fotografía frontal en posición natural de la cabeza (Lundström et al., 1995), para la cual se usó una cámara fotográfica Canon (Canon Digital Rebel XS) según el protocolo de fotografía para la evaluación de la sonrisa presentado por Ackerman \& Ackerman (2002). En cada fotografía frontal se ubicaron los puntos antropométricos: (1) Sn: subnasale, (2) Ls: labiale superius, (3) Sto: stomion, (4) Li: labiale Inferius, (5) Sl: sublabiale, (6) Ch: chelion. Posteriormente se utilizó el software J Image (www.rsbweb.nih.gov/ij/), para determinar las medidas lineales de Grosor del Labio Superior (Sn-Sto), Grosor del bermellon superior (Sto-Ls), Grosor del Labio Inferior (Sto-S1), Grosor del bermellon Inferior (Sto-Li) y Ancho bicomisural (Chd-Chi: chelion derecho-chelion izquierdo). Las medidas tomadas se ilustran en la Figura 1. Las medidas obtenidas fueron calibradas a través de la evaluación clínica directa de 10 individuos al azar usando un calibrador digital de Boley (MP Tools) al 0,01 mm. Para determinar la confiabilidad de las medidas, se repitieron en 10 individuos seleccionados aleatoriamente.

Los datos obtenidos fueron analizados estadísticamente, a través de la prueba U MannWhitney para determinar diferencias entre géneros al establecer el nivel de significancia de $\mathrm{p}<0,05$.

\section{RESULTADOS}

No se detectó diferencia estadísticamente significativa entre laos grupos de medidas aleatorias de 10 individuos. La correlación fue alta para la longitud del labio superior $(\mathrm{r}=0,97)$, la longitud del labio inferior $(\mathrm{r}=0,91)$, la longitud

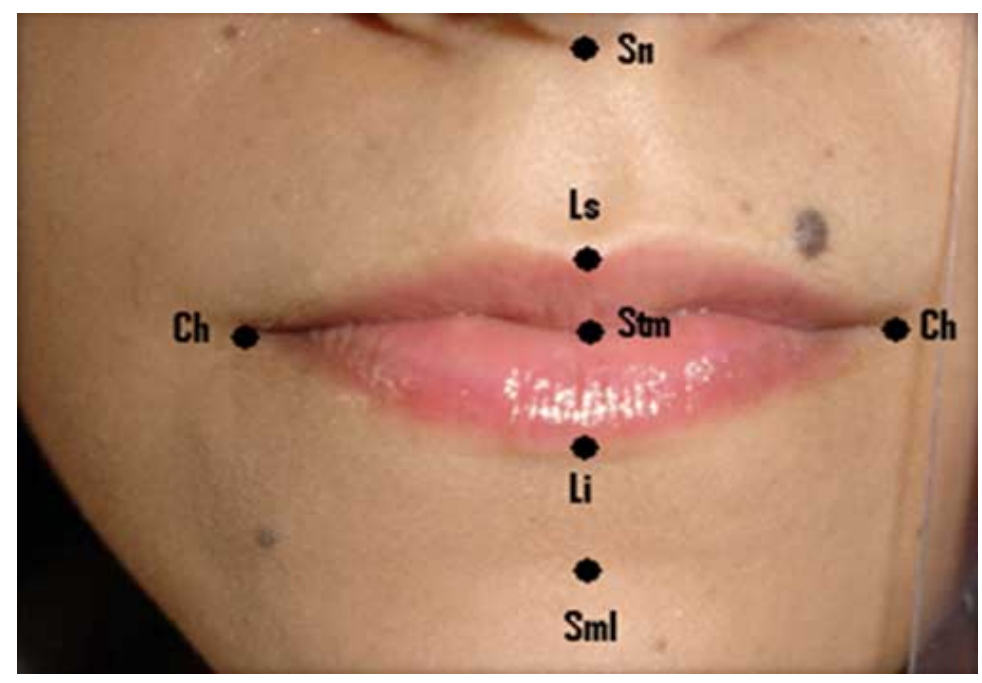

Fig. 1. Puntos antropométricos faciales paradeterminartipo de labio.

del bermellón inferior $(\mathrm{r}=0,90)$, la longitud del bermellón superior y distancia bicomisural $(\mathrm{r}=0,89)$.

La Tabla I muestras las medidas longitudinales promedio para la longitud del labio superior y la longitud del labio inferior. En ninguna de estas medidas se encontraron diferencias estadísticamente significativas entre los dos géneros. Sin embargo, se encontró diferencia estadísticamente significativa $(\mathrm{p}=0,001)$ fue hallada entre los dos géneros en la longitud del bermellón inferior, la longitud del bermellón superior y la distancia bicomisural, el grosor promedio del bermellón superior en las mujeres fue de $23,03 \pm 2,85 \mathrm{~mm}$ y en los hombres fue de $25,86 \pm 2,7 \mathrm{~mm}$. El grosor promedio del bermellón inferior en las mujeres fue de $18,75 \pm 2,42 \mathrm{~mm}$ y en los hombres fue de 20,96 $\pm 2,23 \mathrm{~mm}$. Las medidas mostraron mayores dimensiones labiales en los hombres.

Tabla I. Medidas morfométricas en individuos mestizos según sexo.

\begin{tabular}{|c|c|c|c|c|c|c|}
\hline \multirow[b]{2}{*}{ Medida } & \multicolumn{2}{|c|}{ Mujeres } & \multicolumn{2}{|c|}{ Hombres } & \multirow[b]{2}{*}{$\mathbf{p}$} & \\
\hline & Promedio & DE & Promedio & DE & & \\
\hline Ls-Stms & 7,62 & 1,76 & 7,96 & 2,11 & 0,3891 & NS \\
\hline Ls-Stmi & 11,472 & 1,759 & 11,521 & 1,816 & 0,8932 & NS \\
\hline C-C & 46,901 & 4,829 & 51,198 & 4,208 & 0,0001 & $* * *$ \\
\hline Sn-Ls & 23,03 & 2,85 & 25,86 & 2,70 & 0,0001 & $* * *$ \\
\hline Li-Sml & 18,75 & 2,42 & 20,96 & 2,23 & 0,0001 & $* * *$ \\
\hline
\end{tabular}

\section{DISCUSIÓN}

Los labios son componentes esenciales de la simetría facial y de la estética (Jacono \& Quatela, 2004). Existen pocos estudios que evalúan las características morfológicas de los labios de individuos latinoamericanos. La revisión de la literatura de los últimos 25 años no muestra resultados en esta materia en individuos mestizos colombianos. El presente estudio mostró diferencias en dimorfismo sexual 
en una de las medidas longitudinales de los labios de individuos mestizos colombianos.

La mayoría de los estudios existentes corresponden a estudios transversales como el nuestro y sus resultados concuerdan con el nuestro con respecto al dimorfismo sexual (Ferrario et al.). Sawyer et al. encontraron, a través de un estudio estereofotogramétrico, diferencias entre hombres y mujeres caucásicos, siendo todas las distancias mayores y estadísticamente significativas en hombres. Resultados similares fueron obtenidos por Sforza et al. (2010) y Sforza \& Ferrario (2010) en individuos caucásicos italianos.
Todas las medidas lineales obtenidas fueron mayores en individuos mestizos colombianos que en individuos caucásicos, tanto en individuos de género masculino como femenino. De manera contraria, Cattoni \& Fernandes encontraron en niños brasileros medidas longitudinales labiales menores a las de niños caucásicos norteamericanos.

En conclusión, existen diferencias de dimorfismo sexual en individuos colombianos mestizos. Según nuestro conocimiento, este es el único estudio de su tipo en una población colombiana.

GALINDO-RAMIREZ, S. M.; VARGAS, J.; ORTIZ, M. A. Morphological study of the lips in a colombian population. Int. J. Morphol., 30(2):422424, 2012.

SUMMARY: The aim of this research was to determine sexual dimorphism in lip characteristic in Colombian male and female population through a photogrammetric study. Frontal facial photographs, in 1 to 1 scale, from 120 Colombian persons without congenital or acquired lip alterations were evaluated. Using frontal landmarks, longitudinal dimensions of Upper Lip Height (Sn-Stms), Vermilion Height of the upper lip (Stms-Ls), Lower Lip Height (Stmi-Sml), Vermilion Height of the lower lip (Stmi-Li) and mouth width (ch-ch). J image software was user for phogrammetric analysis (NIH). For Stadistical analysis U Mann-Whitney test was used with SPSS 17.0, in order to determine sexual dimorphism in lip characteristic. No significant sexual dimorphism was found for vermilion height of the upper lip (Stms-Ls) nor vermillion height of the lower lip (Stmi-Li). Significan sexual dimorphism was found for Upper Lip Height (Sn-Stms), Lower Lip Height (Stmi-Sml) and mouth width (ch-ch). All longitudinal measurements were larger in male than female. There was sexual dimorphism in lip measurements in a Colombian population, when evaluated using phoptogrammetric methods.

KEY WORDS: Lip; Sex dimorphism; Vermillion.

\section{REFERENCIAS BIBLIOGRÁFICAS}

Ackerman, M. B. \& Ackerman, J. L. Smile analysis and design in the digital era. J. Clin. Orthod., 36(4):221-36, 2002.

Cattoni, D. M. \& Fernandes, F. D. M. Medidas antropométricas orofaciais de crianças paulistanas e norte-americanas: estudo comparativo (original title: Anthropometric orofacial measurements of children from Sao Paulo and from North America: comparative study). Pró-Fono R. Atual. Cient., 21(1):25-30, 2009.

Chiandussi, S.; Tappuni, A. R.; Watson, T. F.; White, A.; Escudier, M. P.; Sanderson, J. D. \& Challacombe, S. J. Lip impressions: a new method for monitoring morphological changes in orofacial granulomatosis. Oral Dis., 13(1):93-8, 2007.

Davis, J. P.; Valentine, T. \& Davis, R. E. Computer assisted photoanthropometric analyses of full-face and profile facial images. Forensic Sci. Int., 200(1-3):165-76, 2010.

Edler, R. J.; Wertheim, D.; Greenhill, D. \& Jaisinghani, A. Quantitative use of photography in orthognathic outcome assessment. Br. J. Oral Maxillofac. Surg., 49(2):121-6, 2011.

Ferrario, V. F.; Rosati, R.; Peretta, R.; Dellavia, C. \& Sforza, C. Labial morphology: a 3-dimensional anthropometric study. J. Oral Maxillofac. Surg., 67(9):1832-9, 2009.

Jacono, A. A. \& Quatela, V. C. Quantitative analysis of lip appearance after V-Y lip augmentation. Arch. Facial Plast. Surg., 6(3):172-7, 2004.

Lundström, A.; Lundström, F.; Lebret, L. M. \& Moorrees, C. F. Natural head position and natural head orientation: basic considerations in cephalometric analysis and research. Eur. J. Orthod., 17(2):111-20, 1995.
Peck, S. \& Peck, L. Selected aspects of the art and science of facial esthetics. Semin. Orthod., 1(2):105-26, 1995.

Sawyer, A. R.; See, M. \& Nduka, C. 3D stereophotogrammetry quantitative lip analysis. Aesthetic Plast. Surg., 33(4):497-504, 2009.

Scott, C. R.; Goonewardene, M. S. \& Murray, K. Influence of lips on the perception of malocclusion. Am. J. Orthod. Dentofacial Orthop., 130(2):152-62, 2006

Sforza, C. \& Ferrario, V. F. Three-dimensional analysis of facial morphology: growth, development and aging of the orolabial region. Ital. J. Anat. Embryol., 115(1-2):141-5, 2010.

Sforza, C.; Grandi, G.; Binelli, M.; Dolci, C.; De Menezes, M. \& Ferrario, V. F. Age- and sex-related changes in three-dimensional lip morphology. Forensic Sci. Int., 200(1-3):182.e1-7, 2010.

Wong, W. W.; Davis, D. G.; Camp, M. C. \& Gupta, S. C. Contribution of lip proportions to facial aesthetics in different ethnicities: a three-dimensional analysis. J. Plast. Reconstr. Aesthet. Surg., 63(12):2032-9, 2010.

Dirección para correspondencia:

Mario Alejandro Ortiz

Odontólogo, Especialista en Ortodoncia

Maestría en Ciencias Básicas Médicas

Profesor Auxiliar Departamento de Morfología

Universidad del Valle

Cali - COLOMBIA

Email: mariortiz@yahoo.com

Recibido : 08-09-2011

Aceptado: $29-02-2012$ 